\title{
Similar complication rates for irreversible electroporation and thermal ablation in patients with hepatocellular tumors
}

\author{
Niklas Verloh ${ }^{1}$, Isabel Jensch ${ }^{1}$, Lukas Lürken ${ }^{1}$, Michael Haimerl ${ }^{1}$, Marco Dollinger ${ }^{1}$, \\ Philipp Renner ${ }^{2}$, Philipp Wiggermann ${ }^{3}$, Jens Martin Werner ${ }^{4}$, Florian Zeman ${ }^{5}$, Christian \\ Stroszczynski ${ }^{1}$, Lukas Philipp Beyer ${ }^{1}$
}

\author{
${ }^{1}$ Department of Radiology, University Hospital Regensburg, Germany \\ ${ }^{2}$ Department of Surgery, Robert-Bosch-Hospital, Stuttgart, Germany \\ ${ }^{3}$ Department of Radiology, Hospital Braunschweig, Germany \\ ${ }^{4}$ Department of Surgery, University Hospital Regensburg, Regensburg, Germany \\ ${ }^{5}$ Center for Clinical Trials, University Hospital Regensburg, Regensburg, Germany
}

Radiol Oncol 2019; 53(1): 116-122.

Received 2 November 2018

Accepted 22 January 2019

Correspondence to: Lukas Philipp Beyer, M.D., Department of Radiology, University Hospital Regensburg, 93053 Regensburg, Germany. Phone: +49 941944 17464; E-mail: lukas@lukasbeyer.com

Disclosure: No potential conflicts of interest were disclosed.

Background. To compare the frequency of adverse events of thermal microwave (MWA) and radiofrequency ablation (RFA) with non-thermal irreversible electroporation (IRE) in percutaneous ablation of hepatocellular carcinoma (HCC).

Patients and methods. We retrospectively analyzed 117 MWA/RFA and 47 IRE procedures (one tumor treated per procedure; 144 men and 20 women; median age, 66 years) regarding adverse events, duration of hospital and intensive care unit (ICU) stays and occurrence of a post-ablation syndrome. Complications were classified according to the Clavien \& Dindo classification system.

Results. $70.1 \%$ of the RFA/MWA and $63.8 \%$ of the IRE procedures were performed without complications. Grade I and II complications (any deviation from the normal postinterventional course, e.g., analgesics) occurred in 26.5\% (31/117) of MWA/RFA and 34.0\% (16/47) of IRE procedures. Grade III and IV (major) complications occurred in 2.6\% (3/117) of MWA/RFA and $2.1 \%$ (1/47) of IRE procedures. There was no significant difference in the frequency of complications ( $p=0.864$ ), duration of hospital and ICU stay and the occurrence of a post-ablation syndrome between the two groups. Conclusions. Our results suggest that thermal (MWA and RFA) and non-thermal IRE ablation of malignant liver tumors have comparable complication rates despite the higher number of punctures and the lack of track cauterization in IRE.

Key words: tumor ablation; interventional oncology; complications; adverse events; RFA; MWA; IRE

\section{Introduction}

Percutaneous ablation of HCC under imaging control in focal tumor therapy has gained in importance in recent years. Besides surgical resection and liver transplantation, ablation is one of three curative therapies for the treatment of hepatocellular carcinoma (HCC). ${ }^{1}$ Most ablation techniques are so-called thermal methods, which are based on the generation of coagulation necrosis by heat. While radiofrequency ablation (RFA) and microwave ablation (MWA) are among these thermal techniques, irreversible electroporation (IRE), along with other therapies such as electrochemotherapy (ECT), is a non-thermal method for destruction of tumor tissue. . $^{2,3}$ 
IRE uses ultrashort but strong electric fields to create nanopores in the cell membrane, thereby disturbing the cellular homeostasis and subsequently causing cell death by apoptosis. ${ }^{4}$ IRE is not affected by the "heat-sink-effect" ${ }^{\text {"5-9 }}$ and can be applied in close proximity to temperaturesensitive structures such as bile ducts. Therefore, IRE is a useful technique in particular for central liver tumors in the vicinity of larger vessels and bile ducts. ${ }^{2}$ However, IRE has the theoretical disadvantage compared to thermal procedures that up to six electrodes have to be put in place (i.e., multiple trauma by puncture), and the puncture channel cannot be cauterized. First studies with limited number of cases have described possible complications after IRE, such as bleeding, portal vein thrombosis, infections, needle tract seeding and bile duct injuries. ${ }^{10-12}$ However, larger cohort studies and systematic meta-analysis involving more than 10,000 patients exist only for thermal ablation methods. ${ }^{13,14}$ To the best of our knowledge, no work systematically compares so far the complication rates between the thermal methods RFA/MWA and IRE.

This study aims to identify possible differences between complication rates and the morbidity of patients following thermal and non-thermal ablation procedures in order to guide optimal therapy decisions in the future.

\section{Patients and methods}

\section{Study design and participant selection}

The retrospective single-center study was approved by the Ethics Committee of the University Regensburg. It was carried out in accordance with the relevant guidelines and regulations, and informed consent was waived.

The clinical reports of all patients who underwent percutaneous tumor ablation at our interventional oncology center between 07/2010 and 05/2017 were reviewed. The indication for the percutaneous ablation was based exclusively on clinical criteria. Ablations in which more than one tumor was treated were excluded from further examination to ensure comparability between the groups.

The following parameters were assessed for both groups:

- intervention time

- length of hospital stay

- occurrence and length of intensive care unit (ICU) stay

- clinical course post intervention.

\section{Ablation procedures and imaging}

All procedures were performed under general anesthesia and percutaneously under CT fluoroscopy guidance (CAREVision, SOMATOM Sensation 16, Siemens Healthcare).

Hematologic management was performed according to the consensus guidelines of the society of interventional radiology for procedures with a moderate risk of bleeding. ${ }^{15}$ In summary, the INR was corrected to $<1.5$ and the aPTT to values $>1.5$. If the platelets were less than 50,000 per $\mathrm{ml}$, a transfusion with platelet concentrates was performed. Clopidogrel was paused 5 days before ablation. For low molecular weight heparins, the last administration was suspended before the intervention.

IRE procedures were performed with the NanoKnife ${ }^{\circledR}$ System (Angiodynamics ${ }^{\circledR}$ ) using up to six 19G electrodes. The parameters of IRE ablation were as follows: voltage, 1650 to $3000 \mathrm{~V}$; pulse length, $90 \mu \mathrm{s}$; pulses per cycle, 70 . Microwave ablation was carried out using the Acculis microwave tissue ablation system and a $14 \mathrm{G}$ applicator (AngioDynamics, Latham, NY, USA), which operates at $2.45 \mathrm{GHz}$ with a maximum power output of 140 W. For radiofrequency ablation, the StarBurst $\odot$, RITA $\odot$, 1500X ablation system (AngioDynamics, Latham, NY, USA) with monopolar perfused 14G electrodes (StarBurst $\odot$, Talon Semi-Flex, AngioDynamics, Latham, NY, USA) was employed.

After probe placement for the ablation procedure, the parameters were adjusted depending on tumor size with the aim to gain a preferable safety margin of $1 \mathrm{~cm}$.

The first follow-up imaging was routinely conducted on the first working day after the ablation through an abdominal CT and magnetic resonance imaging (MRI). Laboratory tests and physical examinations were continued until discharge of the patient.

\section{Complications}

Complications were documented for each ablation session by evaluating medical records and radiologic images. Since during the post-interventional clinical course the patients were admitted on a surgical ward, post-interventional complications were defined according to the "Classification of Surgical Complications" according to Clavien \& Dindo"16, which allows a detailed insight into the grade of recorded events and even small treatment adjustments are included. 
TABLE 1. Patient and tumor characteristics of the RFA/MWA and IRE group

\begin{tabular}{|c|c|c|c|}
\hline & RFA/MWA $(n=117)$ & IRE $(n=47)$ & p-value \\
\hline Male, n (\%) & $98(83.8)$ & $46(97.9)$ & 0.013 \\
\hline \multicolumn{4}{|l|}{ Age } \\
\hline median (IQR) & $66(14)$ & $71(15)$ & 0.239 \\
\hline range & $45-82$ & $45-83$ & \\
\hline Child-Pugh-Score, n (\%) & & & 0.892 \\
\hline A & $76(65.0)$ & $30(63.8)$ & \\
\hline B & $41(35.0)$ & $17(36.2)$ & \\
\hline BCLC, n (\%) & & & 0.412 \\
\hline A & $68(58.1)$ & $24(51.1)$ & \\
\hline B & $49(41.9)$ & $23(48.9)$ & \\
\hline Tumor size, mm (mean +- SD) & $22+-9$ & $20+-8$ & 0.186 \\
\hline Liver periphery, n (\%) & $85(72.6)$ & $25(53.2)$ & 0.017 \\
\hline Close to a major vessel, n (\%) & 19 (16.2) & 21 (44.7) & $\leq 0.001$ \\
\hline
\end{tabular}

$B C L C=$ Barcelona Clinic Liver Cancer staging system; IQR = interquartile range; IRE = non-therma irreversible electroporation; RFA/MWA = radiofrequency ablation / microwave ablation

TABLE 2. Intervention durations, hospital and intensive care unit (ICU) stay, occurrence of post-ablation syndrome for thermal ablation and non-thermal irreversible electroporation (IRE)

\begin{tabular}{lllll}
\hline & All (164) & $\begin{array}{l}\text { RFA/MWA } \\
(\mathbf{n}=117)\end{array}$ & $\begin{array}{l}\text { IRE } \\
(\mathbf{n}=47)\end{array}$ & p-vale \\
\hline $\begin{array}{l}\text { Intervention duration, minutes } \\
\text { (IQR) }\end{array}$ & $113(96)$ & $103(103)$ & $142(88)$ & 0.031 \\
$\begin{array}{l}\text { Hospital Stay, days } \\
\text { days, median (IQR) }\end{array}$ & $5(4)$ & $5(4)$ & $5(4)$ & 0.752 \\
days, range & $2-50$ & $2-50$ & $2-20$ & \\
ICU Stay, n (\%) & $17(10)$ & $14(12)$ & $3(6)$ & 0.302 \\
days, median (IQR) & $4(5)$ & $4(4)$ & $6(-)$ & 0.222 \\
days, range & $1-9$ & $1-9$ & $3-9$ & \\
Post-ablation Syndrome, n (\%) & $28(17)$ & $21(18)$ & $7(15)$ & 0.607 \\
\hline
\end{tabular}

$I Q R$ = interquartile range; RFA/MWA = radiofrequency ablation / microwave ablation

\section{Post-ablation syndrome}

The post-ablation syndrome is characterized by fever or flu-like symptoms following ablation treatment. ${ }^{17}$ In addition, nausea, vomiting, malaise and body aches have been described. ${ }^{18}$ However, in most cases the symptoms are per se self-limiting under purely symptom-oriented therapy..$^{19}$ In rare cases after treatment of large tumor areas, the postablation syndrome can last up to three weeks. ${ }^{19}$ By contrast, persistent or later onset of fever may indicate a simultaneous infection or abscess. ${ }^{17}$

\section{Statistical analysis}

All statistical analyses were performed with IBM SPSS Statistics (version 24, Chicago, IL, USA) and $R$ 3.2.1. The data are presented as the median with the interquartile range (IQR) if not stated otherwise. The non-parametric Mann-Whitney test was used to analyze patients and tumor characteristics, the intervention time, and the length of the hospital and ICU stay. We used the Chi-Square Test of independence for comparing the observed frequencies of events (ICU-stay $(\mathrm{y} / \mathrm{n})$, post-ablation syndrome $(\mathrm{y} / \mathrm{n}))$ and examined trend of complication grades between both groups using the Cochran-Armitage test for trend. The Fisher's exact test was used to compare the in-house mortality. All tests were two-sided, and values of $\mathrm{p}<0.05$ indicated a significant difference.

\section{Results}

\section{Patient and tumor characteristics}

In this study, 117 patients with HCC were treated with thermal ablations (microwave and radiofrequency ablation) and 47 with irreversible electroporation (IRE). Of the 164 patients, 31 (18.9\%) were listed for liver transplantation and ablation was performed for bridging. The patient and lesion aspects are summarized in Table 1.

A tumor was defined as close to a major vessel if the distance to a vessel with a diameter $>5 \mathrm{~mm}$ was less than $5 \mathrm{~mm}$. A tumor was defined as peripheral if the maximum distance between all parts of the tumor and the liver capsule was $3 \mathrm{~cm}$.

\section{Intervention time}

The median intervention duration of all interventions was $113 \mathrm{~min}$ (IQR 96) (Table 2). A significant difference $(p=0.047)$ was observed between the duration of thermal ablation (103, IQR 103) and irreversible electroporation (142, IQR 88).

\section{Hospital and ICU-stay}

Duration of hospital stays ranged between 2 and 50 days for thermal ablation and between 2 and 20 days for irreversible electroporation, with a median of 5 days (IQR 4 ) for both types of interventions $(p=0.752)$. There was no significant difference between thermal ablation and irreversible electroporation regarding the frequencies $(12.0 \%(14 / 117)$ vs. $6.4 \%(3 / 47), p=0.302)$ as well as the length of ICU-stays with a median ICU stay of 4 days (IQR 4 ) 
TABLE 3. Clavien \& Dindo classification system with the corresponding number of events in the thermal and non-thermal irreversible electroporation (IRE) groups

\begin{tabular}{|c|c|c|c|c|}
\hline \multicolumn{2}{|c|}{ Clavien-Dindo Classification } & \multirow{2}{*}{$\begin{array}{l}\text { SIR Classification } \\
2017\end{array}$} & \multirow{2}{*}{$\begin{array}{l}\text { RFA/MWA } \\
(\mathrm{n}=117)\end{array}$} & \multirow{2}{*}{$\begin{array}{c}\text { IRE } \\
(n=47)\end{array}$} \\
\hline Grade & Description & & & \\
\hline 0 & No complication & No complication & $82(70.1 \%)$ & $30(63.8 \%)$ \\
\hline III & requiring surgical, endoscopic or radiological intervention & & & \\
\hline IIla & intervention not under general anesthesia & Moderate & $1(0.9 \%)$ & 0 \\
\hline Illb & intervention under general anesthesia & Severe & $1(0.9 \%)$ & $1(2.1 \%)$ \\
\hline IV & $\begin{array}{l}\text { Life-threatening complication (including CNS complications) requiring IC/ICU- } \\
\text { management }\end{array}$ & & & \\
\hline
\end{tabular}

$I C U$ = intensive care unit; $I Q R$ = interquartile range; RFA/MWA = radiofrequency ablation / microwave ablation; SIR = Society of Interventional Radiology

for patients treated with thermal ablation versus 6 days for patients treated with IRE $(p=0.222)$.

\section{Clinical course post intervention}

In $17.1 \%$ of the treatments, a post-ablation syndrome occurred during the post-interventional clinical course. Significant differences between thermal ablation (17.9\%) and IRE (14.9\%) were not observed $(\mathrm{p}=0.607)$.

$68.3 \%$ of the procedures were performed without any complication. There was no significant difference between thermal ablation and IRE regarding the occurrence and the severity of a complication $(\mathrm{p}=0.864)$.

One patient died 11 days after MWA due to multiorgan failure after an accidental puncture of the pericardium with hemopericardium and surgical overstitching (Table 3). The incidence of all documented complications is listed in Table 2 . The most common complications of grade II were: blood transfusions $(n=10)$, infections $(n=6)$, electrolyte shifts $(n=4)$.

Grade III complications were: bleeding after RFA followed by angiography without detectable source of bleeding and active monitoring on the ward ( $n=1$; Figure 1), pleural drainage for reactive pleural effusion after sub-diaphragmatic MWA $(n=$
1), HB drop after IRE with explorative laparotomy without intraoperatively visible bleeding source $(\mathrm{n}=1)$. There was one grade IVb complication with partial main portal vein thrombosis after MWA followed by an ICU stay and multi-organ dysfunction (Figure 2).

Primary efficacy, i.e. the percentage of target tumors successful eradicated in the 6-week follow-up imaging, was $84.3 \%$ for RFA/MWA and $67.2 \%$ for IRE.

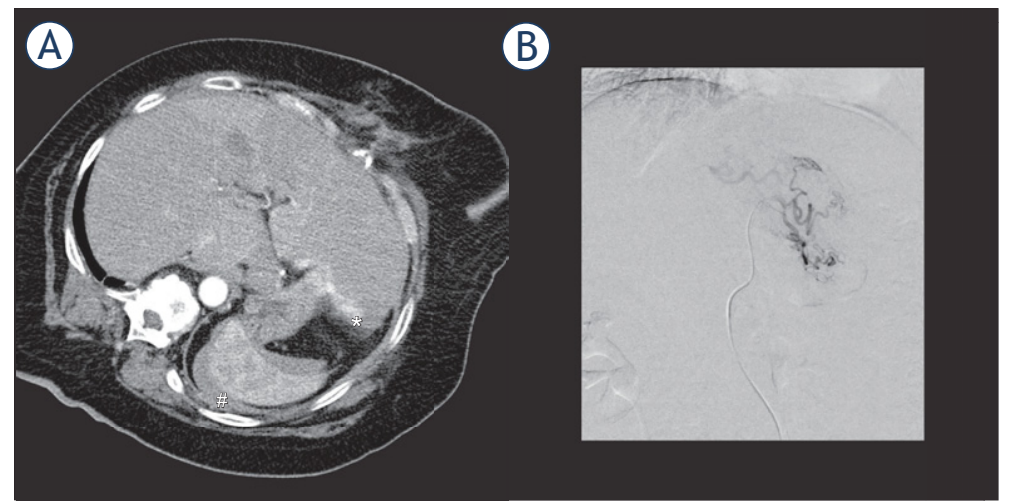

FIGURE 1. 68-year-old female patient with active bleeding (A) on the dorsal side of the left lobe of the liver $\left({ }^{*}\right)$ immediately after RFA and perisplenic hematoma (\#). Diagnostic angiography (B) immediately after the RFA did not show any active arterial bleeding. The patient was then monitored on a normal ward, and the bleeding ceased without further intervention. 


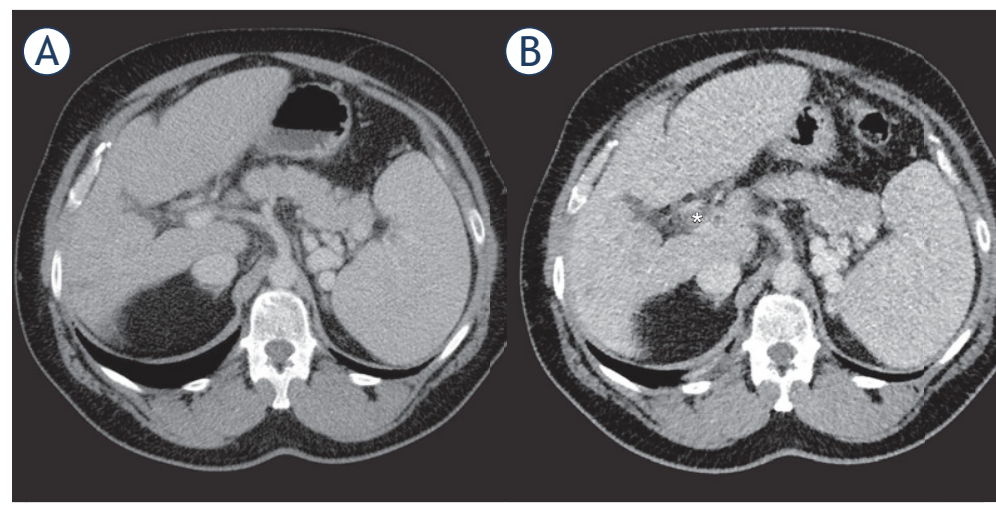

FIGURE 2. 57-year-old male patient with partial thrombosis $(*)$ of the main portal vein one day after microwave ablation (B). (A) Pre-interventional CT scan without visible thrombosis. Anticoagulation led to the regression of thrombosis without any necessary intervention.

\section{Discussion}

Hepatocellular carcinoma (HCC) is the world's third most common cancer leading to death..$^{20}$ In Germany, cancer of the liver and intrahepatic bile ducts are among the top 10 deaths from cancer. ${ }^{21}$ At the time of initial diagnosis, only $10-25 \%$ of patients represent candidates for surgery. 22

Since early detection of cancer enables better prognosis and longer survival, it is recommended that people with the appropriate risk profile be screened for HCC. ${ }^{23}$ According to EASL guidelines, half-yearly ultrasound examinations should be performed in patients with cirrhosis of the liver in stages Child-A and Child-B as well as in patients in stage Child-C if they are eligible for liver transplantation. In the case of abnormalities, additional contrast-enhanced imaging should be performed. . $^{24,25}$

In recent years, such observation strategies of patients with an increased risk-profile led to an earlier diagnosis of the disease and detection of HCCs at earlier stages. In addition to the higher chance of therapeutic response, early-stage patients also benefit from a broader range of different treatment options. ${ }^{26}$

Therapeutic options for hepatocellular carcinoma include surgical resection, liver transplantation, locoregional treatments, and chemotherapy. The final treatment decision depends mainly on the tumor stage, the patient performance status, and the functional liver reserve and requires a multidisciplinary approach. ${ }^{26}$

Which therapy is used in the respective case depends in particular on how far the disease has pro- gressed at the time of diagnosis and in what condition the liver is. The patient's age and the general state of health are also considered when choosing a treatment method..$^{27-29}$ The effectiveness of the local-ablative therapy procedures could be proven in numerous studies of liver cancer up to a size of 3-5 $\mathrm{cm}$. The use of local ablative procedures does not exclude subsequent surgical therapy. Furthermore, local-ablative procedures are often used to bridge waiting time until liver transplantation.

Irreversible Electroporation (IRE) is a novel, minimally invasive technique for the targeted destruction of cells by strong, locally limited electrical fields. Experience to date suggests that, in contrast to other local treatment methods, this technique explicitly targets the tumor cells. Other structures such as blood vessels and bile ducts are not permanently damaged. Potential disadvantages of the IRE are an increased risk of bleeding, due to the necessity of placing up to 6 electrodes in the liver and the lack of the possibility to cauterize the puncture channel.

Considering ongoing discussions which therapy should be used in respective patients, we have analyzed thermal ablation and irreversible electroporation ablation of HCC in the context of postinterventional morbidity.

In our patient cohort, the treatment decision was made by the local tumor board consisting of at least one certified hepatologist, oncologist, intervention radiologist and a highly qualified hepatobiliary surgeon with experiences in liver transplantation.

The incidence rates of the post-ablation syndrome vary significantly between studies. For example, in a prospective study of 54 patients treated with microwave ablation in liver tumors, $60 \%$ developed post-ablation syndrome. ${ }^{30}$ All in all, postablation syndrome is seen in about one-third of patients after thermal ablation ${ }^{18,31}$. In our study, $17 \%$ of the patients had a post-ablation syndrome during the post-interventional clinical course with no differences between thermal and non-thermal ablations.

In this retrospective analysis, we report a systematical comparison between the complication rates of thermal ablation and IRE for a large patient collective.

Livraghi et al. reported six deaths $(0.3 \%)$ and 50 other serious complications (2.2\%) in a multicenter study in which more than 3,500 liver tumors were treated in 2,320 patients by thermal ablation. ${ }^{32}$ Frühling et al. reported in a single-center study a minor complication rate of $20 \%$ (6 out of 30 ) and one $(3.3 \%)$ major complication (bile duct dilata- 
tion and stricture of the portal vein and bile duct) for IRE ${ }^{33}$, while Zeng et al. reports an event rate of $71.4 \%$ of minor and $7.1 \%$ of major complications for a small patient collective $(n=14){ }^{34}$

Comparing our results to previous studies we report a comparable rate of major complications (Grade III-V) for each procedure. However, numbers of minor complications (Grade I and II) are higher compared to previously reported studies. Of note, we used the "Classification of Surgical Complications" according to Clavien \& Dindo". ${ }^{16}$ In comparison to the standardized grading system of the Society of Interventional Radiology (SIR) ${ }^{19,35}$, this classification scheme offers a more detailed insight into the grade of recorded complications and especially includes events as Grade I complications that are not included in the SIR-classification. The SIR-classification only differentiates between major and minor complications; however, the classification reported in this study distinguishes five grades of complications, ranging from minor variation from the normal postinterventional course to the death of a patient.

Despite the retrospective collection of data, we were able to demonstrate that there was no significant difference in the occurrence as well as the grade of complications regarding the use of thermal versus non-thermal ablation, indicating a noninferiority of IRE in comparison to thermal ablation techniques. This is all the more remarkable because the tumors treated with IRE were significantly more frequently close to a major vessel and thus more complication-prone. In contrast, the tumors treated with RFA/MWA were significantly more frequently located in the easily accessible periphery.

We propose that the fear of a higher complication rate should not interfere with the treatment decision regarding thermal versus non-thermal ablation.

\section{References}

1. Kokudo N, Hasegawa $\mathrm{K}$, Akahane $\mathrm{M}$, Igaki $\mathrm{H}$, Izumi $\mathrm{N}$, Ichida $\mathrm{T}$, et al. Evidence-based Clinical Practice Guidelines for Hepatocellular Carcinoma: The Japan Society of Hepatology 2013 update (3rd JSH-HCC Guidelines). Hepatol Res 2015; 45. doi: 10.1111/hepr.12464.

2. Rubinsky B, Onik G, Mikus P. Irreversible electroporation: a new ablation modality-clinical implications. Technol Cancer Res Treat 2007; 6: 37-48. doi: $10.1177 / 153303460700600106$

3. Djokic M, Cemazar M, Popovic P, Kos B, Dezman R, Bosnjak M, et al. Electrochemotherapy as treatment option for hepatocellular carcinoma, a prospective pilot study. EJSO 2018; 44: 651-7. doi: 10.1016/j. ejso.2018.01.090

4. Davalos RV, Mir L, Rubinsky B. Tissue ablation with irreversible electroporation. Ann Biomed Eng 2005; 33: 223. doi: 10.1007/s10439-005-8981-8
5. Patterson EJ, Scudamore $\mathrm{CH}$, Owen DA, Nagy AG, Buczkowski AK. Radiofrequency ablation of porcine liver in vivo: effects of blood flow and treatment time on lesion size. Ann Surg 1998; 227: 559-65. PMID: 9563546

6. Rossi S, Garbagnati F, De Francesco I, Accocella F, Leonardi L, Quaretti P, et al. Relationship between the shape and size of radiofrequency induced thermal lesions and hepatic vascularization. Tumori 1999; 85: 128-32. PMID: 10363079

7. Goldberg SN, Hahn PF, Tanabe KK, Mueller PR, Schima W, Athanasoulis CA, et al. Percutaneous radiofrequency tissue ablation: does perfusion-mediated tissue cooling limit coagulation necrosis? J Vasc Interv Radiol 1998; 9(1 Pt 1): 101-11. PMID: 9468403

8. Goldberg SN, Hahn PF, Halpern EF, Fogle RM, Gazelle GS. Radio-frequency tissue ablation: effect of pharmacologic modulation of blood flow on coagulation diameter. Radiology 1998; 209: 761-7. doi: 10.1148/radiology.209.3.9844671

9. Lu DS, Raman SS, Vodopich DJ, Wang M, Sayre J, Lassman C. Effect of vessel size on creation of hepatic radiofrequency lesions in pigs: assessment of the "heat sink" effect. AJR Am J Roentgenol 2002; 178: 47-51. doi: 10.2214/ ajr.178.1.1780047

10. Dollinger $M$, Beyer $L P$, Haimerl $M$, Niessen $C$, Jung $E-M$, Zeman $F$, et al. Adverse effects of irreversible electroporation of malignant liver tumors under CT fluoroscopic guidance: a single-center experience. Diagn Interv Radiol 2015; 21: 471-5. doi: 10.5152/dir.2015.14442

11. Frühling $P$, Nilsson A, Duraj $F$, Haglund $U$, Norén A. Single-center nonrandomized clinical trial to assess the safety and efficacy of irreversible electroporation (IRE) ablation of liver tumors in humans: short to mid-term results. EJSO 2017; 43: 751-7. doi: 10.1016/j.ejso.2016.12.004

12. Distelmaier $M$, Barabasch $A$, Heil $P$, Kraemer NA, Isfort $P$, Keil $S$, et al. Midterm safety and efficacy of irreversible electroporation of malignant liver tumors located close to major portal or hepatic veins. Radiology 2017; 285: 1023-31. doi: 10.1148/radiol.2017161561

13. Liang $\mathrm{P}$, Wang $\mathrm{Y}, \mathrm{Yu} \mathrm{X}$, Dong $\mathrm{B}$. Malignant liver tumors: treatment with percutaneous microwave ablation--complications among cohort of 1136 patients. Radiology 2009; 251: 933-40. doi: 10.1148/radiol.2513081740

14. Bertot LC, Sato M, Tateishi R, Yoshida H, Koike K. Mortality and complication rates of percutaneous ablative techniques for the treatment of liver tumors: a systematic review. Eur Radiol 2011; 21: 2584-96. doi: 10.1007/s00330011-2222-3

15. Patel IJ, Davidson JC, Nikolic B, Salazar GM, Schwartzberg MS, Walker TG, et al. Consensus guidelines for periprocedural management of coagulation status and hemostasis risk in percutaneous image-guided interventions. $J$ Vasc Interv Radiol 2012; 23: 727-36. doi: 10.1016/j.jvir.2012.02.012

16. Dindo D, Demartines N, Clavien PA. Classification of surgical complications: a new proposal with evaluation in a cohort of 6336 patients and results of a survey. Ann Surg 2004; 240: 205-13. doi: 10.1097/01. sla.0000133083.54934.ae

17. Sahay A, Sahay N, Kapoor A, Kapoor J, Chatterjee A. Percutaneous imageguided radiofrequency ablation of tumors in inoperable patients - immediate complications and overall safety. Indian J Palliat Care 2016; 22: 67-73. doi: 10.4103/0973-1075.173951

18. Carrafiello G, Lagana D, lanniello A, Dionigi G, Novario R, Recaldini C et al. Post-radiofrequency ablation syndrome after percutaneous radiofrequency of abdominal tumours: one centre experience and review of published works. Australas Radiol 2007; 51: 550-4. doi: 10.1111/j.14401673.2007.01871.x

19. Goldberg SN, Grassi CJ, Cardella JF, Charboneau JW, Dodd GD, Dupuy DE, et al. Image-guided tumor ablation: standardization of terminology and reporting criteria. J Vasc Interv Radiol 2009; 20(7 Suppl): S377-90. doi: 10.1016/j.jvir.2009.04.011

20. Feher J, Lengyel G. [Hepatocellular carcinoma: occurrence, risk factors, biomarkers]. Orv Hetil 2010; 151: 933-40. doi: 10.1556/oh.2010.28900

21. Statistisches-Bundesamt. Diagnosedaten der krankenhäuser ab 2000 zweigstelle bonn june 2017 [updated 12. 06. 2017]. Available from: http:// www.gbe-bund.de.

22. Vauthey JN, Dixon E, Abdalla EK, Helton WS, Pawlik TM, Taouli B, et al. Pretreatment assessment of hepatocellular carcinoma: expert consensus statement. HPB 2010; 12: 289-99. doi: 10.1111/j.1477-2574.2010.00181.x.

23. Desjardins LA. Hepatocellular carcinoma. Clin J Oncol Nurs 2002; 6: 107-8. doi: 10.1188/02.cjon.107-108 
24. Malek NP, Schmidt S, Huber P, Manns MP, Greten TF. The diagnosis and treatment of hepatocellular carcinoma. Dtsch Arztebl Int 2014; 111: 101-6. doi: 10.3238/arztebl.2014.0101.

25. EASL Clinical Practice Guidelines: Management of hepatocellular carcinoma. J Hepatol 2018; 69: 182-236. doi: 10.1016/j.jhep.2018.03.019

26. Raza A, Sood GK. Hepatocellular carcinoma review: current treatment, and evidence-based medicine. World J Gastroenterol 2014; 20: 4115-27. doi: 10.3748/wjg.v20.i15.4115

27. Bruix J, Sherman M. Management of hepatocellular carcinoma. Hepatology 2005; 42: 1208-36. doi: 10.1002/hep.24199

28. Ryder SD. Guidelines for the diagnosis and treatment of hepatocellular carcinoma (HCC) in adults. Gut 2003; 52 (Suppl 3): iii1-8. doi: 10.1016/j. jvir.2013.09.005

29. Ikai I, Kudo M, Arii S, Omata M, Kojiro M, Sakamoto M, et al. Report of the 18th follow-up survey of primary liver cancer in Japan. Hepatol Res 2010; 40: 1043-59. doi: 10.1111/j.1872-034X.2010.00731.x

30. Andreano A, Galimberti S, Franza E, Knavel EM, Sironi S, Lee FT, et al. Percutaneous microwave ablation of hepatic tumors: prospective evaluation of postablation syndrome and postprocedural pain. J Vasc Interv Radiol 2014; 25: 97-105. e2. doi: 10.1016/j.jvir.2013.09.005

31. Dodd GD, 3rd, Napier D, Schoolfield JD, Hubbard L. Percutaneous radiofrequency ablation of hepatic tumors: postablation syndrome. AJR Am J Roentgenol 2005; 185: 51-7. doi: 10.2214/aj.185.1.01850051

32. Livraghi T, Solbiati L, Meloni MF, Gazelle GS, Halpern EF, Goldberg SN Treatment of focal liver tumors with percutaneous radio-frequency ablation: complications encountered in a multicenter study. Radiology 2003, 226: 441-51. doi: 10.1148/radiol.2262012198

33. Fruhling $P$, Nilsson A, Duraj F, Haglund $U$, Noren A. Single-center nonrandomized clinical trial to assess the safety and efficacy of irreversible electroporation (IRE) ablation of liver tumors in humans: Short to mid-term results. Eur J Surg Oncol 2017; 43: 751-7. doi: 10.1016/j.ejso.2016.12.004

34. Zeng J, Liu G, Li ZH, Yang Y, Fang G, Li RR, et al. The safety and efficacy of irreversible electroporation for large hepatocellular carcinoma. Technol Cancer Res Treat 2017; 16: 120-4. doi: 10.1177/1533034616676445

35. Omary RA, Bettmann MA, Cardella JF, Bakal CW, Schwartzberg MS, Sacks $D$, et al. Quality improvement guidelines for the reporting and archiving of interventional radiology procedures. J Vasc Interv Radiol 2003; 14(9 Pt 2): S293-5. PMID: 12354820 